\title{
COVID-19 in Liver and Kidney Transplant Recipients: An Initial Single-center Experience in Iran
}

\author{
NazaninZahra Sepehri (iD ${ }^{1,2}$, Mehrdad Karajizadeh (iD ${ }^{3,}{ }^{,}$, Reza Nikandish (iD) ${ }^{4}$, Seyed Ali Hosseini (iD) ${ }^{5}$ and \\ Kamran Moazzemi ${ }^{2}$ \\ ${ }^{1}$ School of Medicine, Shiraz University of Medical Sciences, Shiraz, Iran \\ ${ }^{2}$ Shiraz Organ Transplant Center, Avicenna Hospital, Shiraz University of Medical Sciences, Shiraz, Iran \\ ${ }^{3}$ Department of Health Information Management, Health Human Resources Research Center, School of Management \& Medical Information Sciences, Shiraz University of \\ Medical Sciences, Shiraz, Iran \\ ${ }^{4}$ Anesthesiology and Critical Care Research Center, Shiraz University of Medical Sciences, Shiraz, Iran \\ ${ }^{5}$ Student Research Committee, Shiraz University of Medical Sciences, Shiraz, Iran \\ "Corresponding author: Health Human Resources Research Center, Almas Building, Alley 29, Qasrodasht Ave, P.O. Box: 71336-54361, Shiraz, Iran. Tel: +98-71132340774, Email: \\ mehrdad.karaji@gmail.com
}

Received 2021 August 30; Revised 2021 September 06; Accepted 2021 September 10.

Keywords: Liver Transplant, Kidney Transplant, COVID-19, SARS-CoV-2

\section{Introduction}

The Coronavirus Disease 2019(COVID-19) pandemic has immensely affected worldwide, giving rise to significant morbidity and mortality (1). Besides age, underlying disease represents a major risk factor for COVID-19 severity, with kidney and liver transplant recipients being highly susceptible to a severe course of the disease despite not being at a greater risk of infection relative to the general population (1-3). In fact, patients with solid organ transplants have a higher rate of mortality ( 20\%) due to COVID19 than their non-transplant counterparts $(1,4)$.

The clinical presentation and outcome of COVID-19 in solid organ transplant recipients vary widely between patient groups and countries (1). Furthermore, limited data are available about the state of such patients (particularly liver transplant recipients) in Iran, with only a small number of cases having been studied (5-7). Therefore, the present study aimed to shed light on the situation of kidney and liver transplant recipients admitted with COVID-19 in Iran.

\section{Methods}

We report 30 kidney and liver transplant recipients who were admitted to Abu Ali Sina Organ Transplant Center (the largest transplantation referral center in the Middle East) due to COVID-19 between March 20 and October 20, 2020. All kidney/liver transplant adult recipients who had a positive severe acute respiratory syndrome coronavirus 2 (SARS-CoV-2) nasopharyngeal RT-PCR assay and required admission were included. A second SARS-CoV-2 RTPCR assay was performed in our hospital for further confir- mation.

\section{Results and Discussion}

In this cross-sectional study of COVID-19 in kidney and liver transplant recipients, 30 adults with a mean age of $52 \pm 11.75$ years were included. In this population, $76.70 \%$ were males and the remaining patients $23.30 \%$ were females. The mean Body Mass Index (BMI) was $25.38 \pm 3.21$ $\mathrm{kg} / \mathrm{m}^{2}$. The majority of the patients were kidney transplant recipients (70\%), while 30\% were liver transplant recipients.

The mean temperature, heart rate, respiratory rate, systolic blood pressure, diastolic blood pressure, oxygen saturation, and Glasgow Coma Scale (GCS) score were 36.94 $\pm 0.52^{\circ} \mathrm{C}, 92.83 \pm 18.95,18.76 \pm 1.85,129.23 \pm 22.19 \mathrm{mmHg}$, $77.90 \pm 18.33 \mathrm{mmHg}, 89.60 \pm 11.22 \%$, and $14.80 \pm 0.61$, respectively. Notably, four patients had prolonged capillary refill times, three of whom were severely dehydrated. Also, $40 \%$ of the patients required wheelchair assistance at the time of presentation. The comorbidities of the patients prior to admission are represented in Figure 1.

Figure 2 summarizes the frequency of different complications and conditions diagnosed during hospitalization among these patients. Clearly, the most frequent complication was acute kidney injury.

There were nine recorded COVID-19-related deaths in this population during the study period, representing a $30 \%$ in-hospital mortality rate. This included six out of 21 (28.6\%) kidney transplant patients and three out of nine (33.3\%) liver transplant patients. Notably, the majority of these patients experienced acute kidney injury prior to 


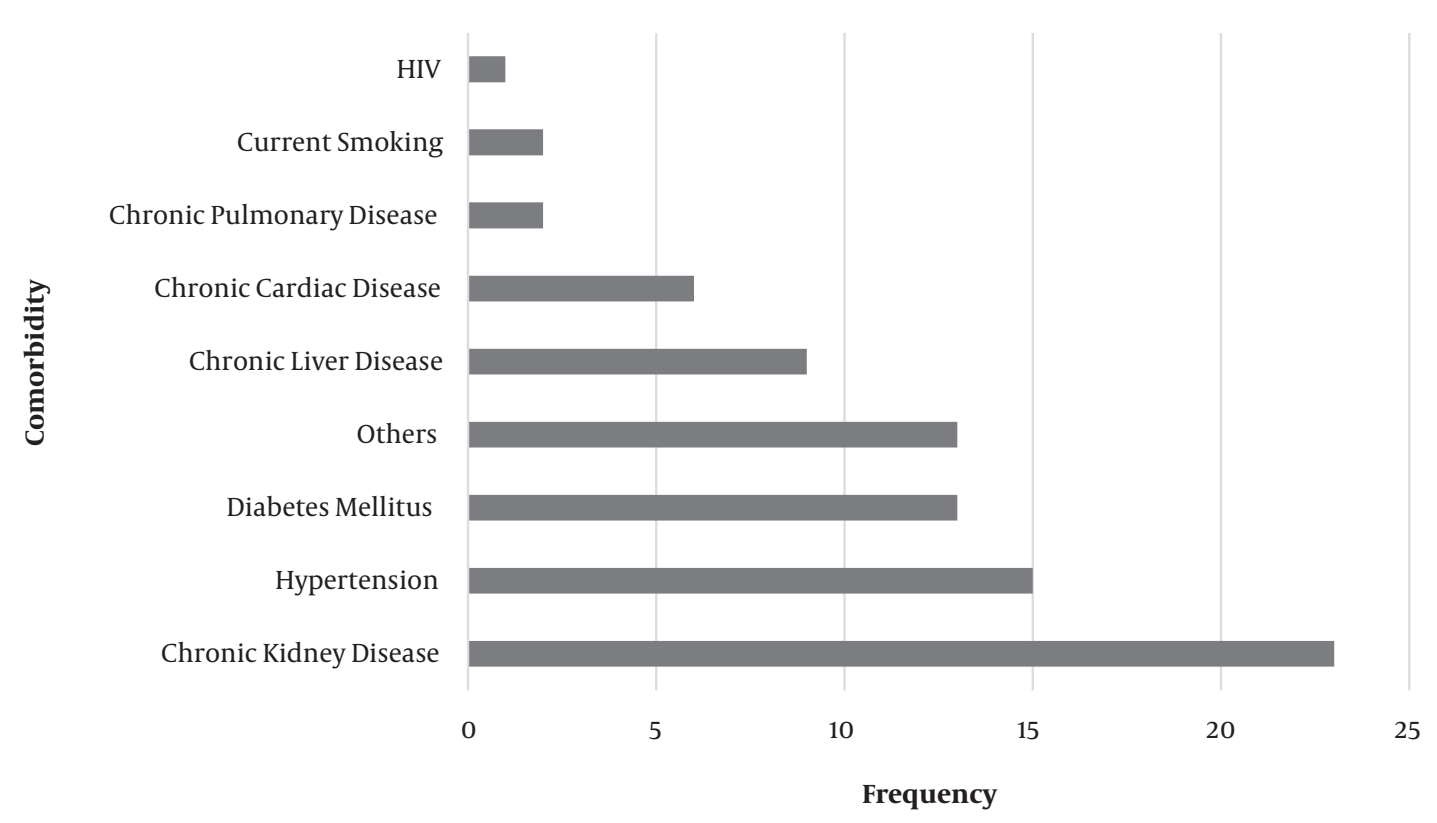

Figure 1. Comorbidities of 30 liver/kidney transplant recipients admitted with COVID-19.

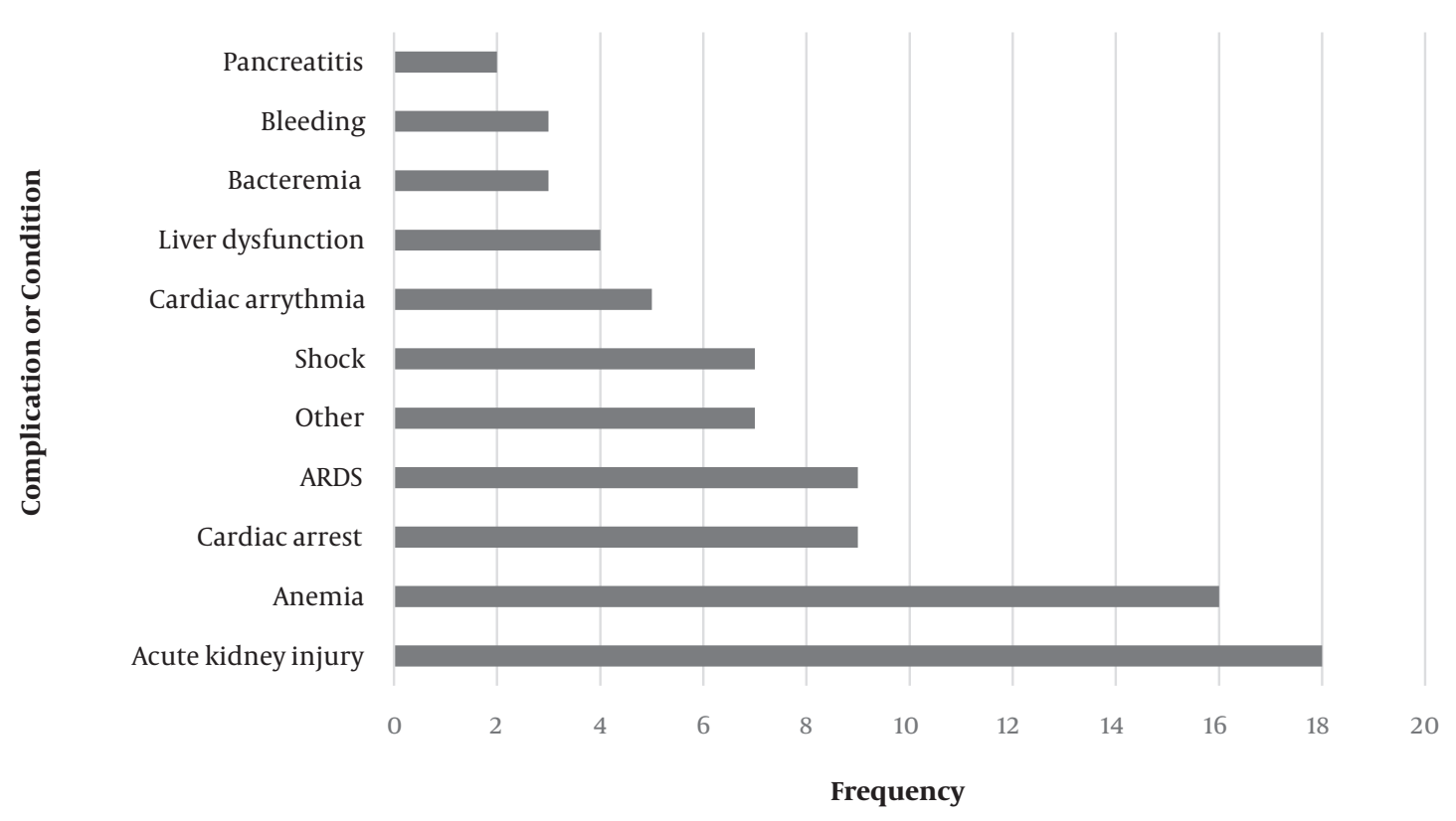

Figure 2. Complications or conditions diagnosed during hospitalization among 30 liver/kidney transplant recipients admitted with COVID-19. ARDS: Acute respiratory distress syndrome. 
their death, including five of the six in the former group and two of the three in the latter group. The remaining 21 patients were discharged, and complete recovery was recorded for them all at the three-month follow-up.

Among our sample population of solid organ transplant patients, the most prominent signs and symptoms were fatigue and shortness of breath (each 73.3\%), followed by fever and headache (each 53.3\%), nausea/vomiting (50\%), myalgia (43.3\%), and cough (40\%). Notably, $36.7 \%$ of our patients had altered consciousness. Compared with reports on general Chinese and Iranian populations, it seems that our patients had a fairly higher rate of shortness of breath and fatigue, which is probably explained by the underlying condition and highlights a more severe disease course in these patients $(8,9)$. Among kidney transplant COVID-19 patients, the most frequently reported symptoms are fever (85\%), dry cough (70\%), myalgia (60\%), and dyspnea (57\%) (10). Variations may be explained by differences in study populations, with our results being limited to those solid organ transplant recipients that required hospitalization.

Among our study population, the most frequent complication was acute kidney injury, which occurred in 60\% of the patients. This is comparable with the reported rate of AKI in the general population of hospitalized COVID19 patients (56.9\%) (11), implying that solid organ transplant recipients are not at a greater risk of AKI than nontransplant recipients. Our result is also comparable with that of the study by Monfared et al., where 12 out of $22(\sim 55 \%)$ kidney transplant recipients with COVID-19 in Rasht, Iran, developed AKI during hospitalization (6). However, a notable finding of our study was that roughly $78 \%$ of the liver or kidney transplant recipients who died due to COVID-19 experienced AKI, compared with a rate of 53\% among those who recovered. This indicates the necessity of paying greater attention to this complication as a marker associated with fatality.

In Iran, the in-hospital mortality rate of COVID-19 has been reported as $24.4 \%$ in a national retrospective cohort study (9). According to the literature, solid organ transplants have a higher rate of mortality $(\sim 20 \%)$ due to COVID19 than their non-transplant counterparts $(1,4)$. This issue was corroborated by our results, where an in-hospital mortality rate of $30 \%$ was recorded, comprising $28.6 \%$ of kidney transplant patients and 33.3\% of liver transplant patients. This is in line with a mortality rate of $27.27 \%$ reported in a similar Iranian study, involving 22 kidney transplant recipients hospitalized due to COVID-19 (6), and is also comparable with the rate of $29.2 \%$ reported among kidney transplant patients in Scotland (12). The slightly higher mortality rate in the Iranian studies relative to some research from other countries involving solid organ transplant recipients with COVID-19 may be explained by differences in healthcare systems. Our relatively high rate of mortality may also be explained by the high mean age ( $52 \pm 11.75$ years) of our study group considering that COVID-19 mortality increases with age (13). Nonetheless, what is clear from our study is that the situation of hospitalized liver and kidney transplant patients afflicted with COVID-19 may be worse in Iran than in other countries, and further efforts must be devised to prevent and, when necessary, manage this disease in this high-risk population group.

It can be concluded that, following the trend described in studies from other countries, liver and kidney transplant patients afflicted with COVID-19 in Iran experience a higher rate of severe disease and mortality than the general population.

\section{Footnotes}

Authors' Contribution: NZS: Study concept and design, data acquisition, data analysis, drafting, and revising the paper. MK: Contributed to conceiving and designing the study, interpreting the data, commenting on drafts, and making significant revisions to the paper. SAH: Contributed to designing the study, interpreting the data, and making significant revisions to the paper. KM:Contributed to designing the study, interpreting the data, and making significant revisions to the paper.

Conflict of Interests: The authors declare no potential conflicts of interest to the research.

Ethical Approval: IR.SUMS.REC.1399.398.

Funding/Support: This research project was supported by the Shiraz University of Medical Sciences (Code No. 9901-122-22458).

Informed Consent: It was not declared by the authors.

\section{References}

1. Fraser J, Mousley J, Testro A, Smibert OC, Koshy AN. Clinical presentation, treatment, and mortality rate in liver transplant recipients with coronavirus disease 2019: A systematic review and quantitative analysis. Transplant Proc. 2020;52(9):2676-83. doi: 10.1016/j.transproceed.2020.07.012. [PubMed: 32891405]. [PubMed Central: PMC7391976].

2. Akdur A, Karakaya E, Ayvazoglu Soy EH, Yarbug Karakayali F, Yildirim S, Torgay A, et al. Liver and kidney transplant during a 6-month period in the COVID-19 pandemic: A single-center experience. Exp Clin Transplant. 2020;18(5):564-71. doi: 10.6002/ect.2020.0388. [PubMed: 33143601].

3. Akdur A, Karakaya E, Ayvazoglu Soy EH, Alshalabi O, Kirnap M, Arslan $\mathrm{H}$, et al. Coronavirus disease (COVID-19) in kidney and liver transplant patients: A single-center experience. Exp Clin Transplant. 2020;18(3):270-4. doi: 10.6002/ect.2020.0193. [PubMed: 32519617].

4. Raja MA, Mendoza MA, Villavicencio A, Anjan S, Reynolds JM, Kittipibul V, et al. COVID-19 in solid organ transplant recipients: A systematic review and meta-analysis of current literature. Transplant Rev 
(Orlando).2021;35(1):100588. doi: 10.1016/j.trre.2020.100588. [PubMed: 33246166]. [PubMed Central: PMC7666542].

5. Molaei H, Khedmat L, Nemati E, Rostami Z, Saadat SH. Iranian kidney transplant recipients with COVID-19 infection: Clinical outcomes and cytomegalovirus coinfection. Transpl Infect Dis. 2021;23(1). e13455. doi: 10.1111/tid.13455. [PubMed: 32881220].

6. Monfared A, Dashti-Khavidaki S, Jafari R, Jafari A, Ramezanzade E, Lebadi MK, et al. Clinical characteristics and outcome of COVID19 pneumonia in kidney transplant recipients in Razi hospital, Rasht, Iran. Transpl Infect Dis. 2020;22(6). e13420. doi: 10.1111/tid.13420. [PubMed: 32681756]. [PubMed Central: PMC7404381].

7. Niknam R, Malek-Hosseini SA, Hashemieh SS, Dehghani M. COVID-19 in liver transplant patients: Report of 2 cases and review of the literature. Int Med Case Rep J. 2020;13:317-21. doi: 10.2147/IMCRJ.S265910. [PubMed: 32801943]. [PubMed Central: PMC7410393].

8. Wan S, Xiang Y, Fang W, Zheng Y, Li B, Hu Y, et al. Clinical features and treatment of COVID-19 patients in northeast Chongqing. J Med Virol. 2020;92(7):797-806. doi: 10.1002/jmv.25783. [PubMed:32198776]. [PubMed Central: PMC7228368].

9. Jalili M, Payandemehr P, Saghaei A, Sari HN, Safikhani H, Kolivand P. Characteristics and mortality of hospitalized patients with COVID-
19 in Iran: A national retrospective cohort study. Ann Intern Med. 2021;174(1):125-7. doi: 10.7326/M20-2911. [PubMed: 32687717]. [PubMed Central: PMC7393802].

10. Nair V, Jandovitz N, Hirsch JS, Nair G, Abate M, Bhaskaran M, et al. COVID-19 in kidney transplant recipients. Am J Transplant. 2020;20(7):1819-25. doi: 10.1111/ajt.15967. [PubMed: 32351040]. [PubMed Central: PMC7267603].

11. Fisher M, Neugarten J, Bellin E, Yunes M, Stahl L, Johns TS, et al. AKI in hospitalized patients with and without COVID-19: A comparison study. J Am Soc Nephrol. 2020;31(9):2145-57. doi: 10.1681/ASN.2020040509. [PubMed: 32669322]. [PubMed Central: PMC7461660].

12. Bell S, Campbell J, McDonald J, O'Neill M, Watters C, Buck K, et al. COVID-19 in patients undergoing chronic kidney replacement therapy and kidney transplant recipients in Scotland: findings and experience from the Scottish renal registry. BMC Nephrol. 2020;21(1):419. doi: 10.1186/s12882-020-02061-8. [PubMed: 33004002]. [PubMed Central: PMC7528715].

13. Woolf SH, Chapman DA, Lee JH. COVID-19 as the leading cause of death in the United States. JAMA. 2021;325(2):123-4. doi: 10.1001/jama.2020.24865. [PubMed: 33331845]. 